\title{
Immunohistochemical studies of pulmonary large cell neuroendocrine carcinoma: A possible association between staining patterns with neuroendocrine markers and tumor response to chemotherapy
}

\author{
Yugo Tanaka, MD, ${ }^{\mathrm{a}}$ Hiroyuki Ogawa, MD, ${ }^{\mathrm{a}}$ Kazuya Uchino, MD, ${ }^{\mathrm{a}}$ Chiho Ohbayashi, MD, ${ }^{\mathrm{b}}$ \\ Yoshimasa Maniwa, MD, ${ }^{\mathrm{c}}$ Wataru Nishio, MD ${ }^{\mathrm{c}}$ Atsunori Nakao, MD, ${ }^{\mathrm{d}}$ and Masahiro Yoshimura, $\mathrm{MD}^{\mathrm{a}}$
}

\begin{abstract}
Objective: Pulmonary large cell neuroendocrine carcinoma is a rare high-grade malignant tumor. Because large cell neuroendocrine carcinoma is rare, the optimal treatment, including perioperative chemotherapy, has not been defined. We retrospectively analyzed the correlation among the effectiveness of perioperative chemotherapy in treating large cell neuroendocrine carcinoma, pathologic stage, and immunoreactivity to neuroendocrine markers.
\end{abstract}

\begin{abstract}
Methods: A total of 63 patients with pulmonary large cell neuroendocrine carcinoma undergoing surgical resection from 2001 to 2009 were included. The resected tumors were immunohistochemically stained with the 3 neuroendocrine markers synaptophysin, chromogranin A, and neural cell adhesion molecule. We categorized patients who were positive for all 3 markers as the triple-positive group and those who were negative for 1 or 2 markers as the non-triple-positive group.
\end{abstract}

\begin{abstract}
Results: Perioperative chemotherapy resulted in better overall survival than surgery alone $(P=.042)$. Multivariate analysis of survival revealed that perioperative chemotherapy was a significant independent prognostic factor (hazard ratio, $0.323 ; 95 \%$ confidence interval, $0.112-0.934 ; P=.0371$ ). Among the patients who received perioperative chemotherapy, the non-triple-positive group had a significantly greater 5-year survival rate than the triple-positive group $(P=.0216)$. Moreover, among the non-triple-positive group, a significantly greater 5 -year survival rate was observed for the patients who underwent surgery with chemotherapy than for those who underwent surgery without chemotherapy $(P=.0081)$. In contrast, no difference was found in 5 -year survival between patients with chemotherapy and those without chemotherapy when the tumors were triple positive.
\end{abstract}

Conclusions: Our results suggest that perioperative chemotherapy might benefit the survival of patients with pulmonary large cell neuroendocrine carcinoma, in particular when the tumors are not immunoreactive to all 3 neuroendocrine markers. (J Thorac Cardiovasc Surg 2013;145:839-46)

Pulmonary large cell neuroendocrine carcinoma (LCNEC), proposed as a separate tumor category by Travis and colleagues ${ }^{1}$ in 1991 , is distinguished from typical carcinoid, atypical carcinoid, and small-cell lung carcinoma (SCLC) by its morphologic and biologic features. In 1999, the World Health Organization classified LCNEC as a variant of large cell carcinoma. ${ }^{2}$ Pulmonary LCNEC represents about $2 \%$ to $3 \%$ of all lung malignancies and is associated

From the Departments of Thoracic Surgery ${ }^{\mathrm{a}}$ and Pathology, ${ }^{\mathrm{b}}$ Hyogo Cancer Center, Akashi City, Hyogo, Japan; Division of Thoracic Surgery, ${ }^{\mathrm{c}}$ Kobe University Graduate School of Medicine, Kobe, Japan; and Department of Surgery, ${ }^{\mathrm{d}}$ University of Pittsburgh Medical Center, Pittsburgh, Pa

Disclosures: Authors have nothing to disclose with regard to commercial support.

Received for publication Nov 13, 2011; revisions received Jan 26, 2012; accepted for publication March 16, 2012; available ahead of print April 12, 2012.

Address for reprints: Masahiro Yoshimura, MD, Department of Thoracic Surgery, Hyogo Cancer Center, 13-70, Kitaouji-cho, Akashi 673-8558, Japan (E-mail: myoshi@hp.pref.hyogo.jp).

0022-5223/ $\$ 36.00$

Copyright (C) 2013 by The American Association for Thoracic Surgery

doi:10.1016/j.jtcvs.2012.03.036 with a worse prognosis than other non-SCLC (NSCLC), even in the early stage. ${ }^{3-6}$ However, in a recent Japanese study with a large sample size, Asamura and colleagues ${ }^{7}$ reported that no prognostic difference was found between pulmonary LCNEC and SCLC.

Several small-scale retrospective studies have demonstrated that perioperative chemotherapy could improve the survival of patients with pulmonary LCNEC. Perioperative chemotherapy is recommended even for patients with resectable stage I LCNEC because of its aggressive course, remarkably dismal prognosis, and high potential for metastasis. ${ }^{8-11}$ However, owing to the rarity of this tumor, the incidence, prognosis, and optimal treatment remain to be determined.

In the present study, we retrospectively analyzed the efficacy of perioperative chemotherapy in treating pulmonary LCNEC. Furthermore, we examined the correlation between the sensitivity of LCNEC and perioperative chemotherapy and the immunohistochemical staining patterns of 


$$
\begin{aligned}
& \text { Abbreviations and Acronyms } \\
& \text { CPT-11 }=\text { irinotecan } \\
& \text { LCNEC }=\text { large cell neuroendocrine carcinoma } \\
& \text { NCAM }=\text { neural cell adhesion molecule } \\
& \text { NSCLC }=\text { non-small-cell lung carcinoma } \\
& \text { SCLC }=\text { small-cell lung carcinoma } \\
& \text { VP-16 }=\text { etoposide }
\end{aligned}
$$

the tumors with 3 immunohistochemical neuroendocrine markers, synaptophysin, chromogranin A, and neural cell adhesion molecule (NCAM). Although our experiences in 2 institutions do not allow us to reach a definite conclusion owing to the small number of subjects, the present preliminary study may be useful in generating a hypothesis to determine the immunohistochemical biomarkers to predict LCNEC's response to perioperative chemotherapy in future prospective multi-institutional trials.

\section{METHODS}

We retrospectively examined the clinical data of 63 patients with pulmonary LCNEC who underwent complete surgical resection from 2001 to 2009. All follow-up data were current as of December 31, 2011. All patients who underwent surgery in 2009 were included in the present study, because more than 2 years have passed since their surgery. The median follow-up period was 32.3 months (range, 2.8-95.3 months). The Hyogo Cancer Center and Kobe University Hospital institutional review boards approved the study, and each participant provided informed consent. LCNEC was diagnosed using the following histopathologic criteria: (1) neuroendocrine morphology such as an organoid, palisading, rosette-like, or trabecular growth pattern; (2) high mitotic count $(\geq 11 / 10$ high-power fields [HPF]); (3) tumor necrosis (often large zone); (4) large cell size with low nuclear/cytoplasmic ratio, vesicular or fine chromatin, and/or frequent nucleoli; and (5) positive immunostaining for 1 or more of the neuroendocrine markers, synaptophysin, chromogranin A, and NCAM. ${ }^{2}$

Immunohistochemical stains were performed on 4-mm-thick, formalinfixed, paraffin-embedded sections. The deparaffinized sections underwent $\mathrm{CC} 1$ buffer pretreatment $(\mathrm{pH} 8.5$, ethylenediaminetetraacetic acid; Roche, Mannheim, Germany) and were immunostained for the markers with the streptavidin-biotin technique with an automated immunostainer (Benchimark; Ventana, Tucson, Ariz) according to the manufacturer's instructions. Antibodies against chromogranin A (polyclonal, 1:500 dilution; Dako, Glostrup, Denmark), synaptophysin (monoclonal, clone 27G12, 1:2 dilution; Nichirei, Tokyo, Japan), CD56 (NCAM; monoclonal, 1:100 dilution; Novocastra, Newcastle, UK), and Ki-67 (monoclonal 1:100 dilution; Dako) were used.

All samples were evaluated by an expert pathologist (C.O.) without knowledge of the patient's outcome. Plural sections, more than 10 sections in most cases, were prepared in each case, and 1 representative specimen involving tumor was selected for immunohistochemistry. The final results were reported as negative (no positive cells) or positive (immunoreactive). Proliferative activity was expressed as the MIB-1 index, which was calculated as the proportion of Ki-67-positive cells by counting 500 to 1000 cancer cells. The mitotic counts were performed using an Olympus BX53 microscope at a magnification of $\times 400$, counting 3 sets of 10 HPF for each tumor. The area with the greatest numbers of mitoses was counted. In the present study, we included pure LCNEC and combined LCNEC, in which at least 1 portion of neuroendocrine differentiation or morphology in NSCLC was LCNEC. The medical records provided information on the patient age, gender, smoking status, pathologic stage, perioperative chemotherapy, and operative procedure. The determination of disease stage was based on the TNM classification using the International Union Against Cancer staging system. ${ }^{12}$

We classified patients into 2 groups to investigate the correlation between the sensitivity of LCNEC to perioperative chemotherapy and the results of immunohistochemical staining of neuroendocrine markers. We categorized the patients who were positive for all 3 neuroendocrine markers (ie, synaptophysin, chromogranin A, and NCAM) as the triplepositive group and those who were negative for 1 or 2 of the markers as the non-triple-positive group. Statistical analyses were performed using JMP, version 8, software (SAS Institute, Cary, NC). Student's $t$-test and the chi-square test were performed to assess the significance of the differences in age, gender, smoking status, surgical procedure, and pathologic stage between the triple-positive and non-triple-positive groups. Survival was calculated using the Kaplan-Meier method, and differences in the distributions were evaluated using the log-rank test. The Cox proportional hazards model was used to evaluate the association between the prognostic factors and survival rate after pulmonary resection, with the hazards ratio and $95 \%$ confidence intervals. Significance was set at $P<.05$.

\section{RESULTS}

The clinicopathologic characteristics of the 63 patients with pulmonary LCNEC who underwent surgical resection are listed in Table 1 . The patient age ranged from 30 to 84 years (mean age, 67.0 years). Of the 63 patients, $54(87 \%)$ were men, and $58(92 \%)$ were former or current smokers. The surgical procedures included 55 lobectomies, 2 segmentectomies, and 6 wedge resections with complete resection (R0). Of the 55 lobectomies, 8 bronchoplastic procedures were performed and 6 extended resections were required because of tumor invasion into the adjacent tissue, including muscle and rib $(\mathrm{n}=3)$, parietal pleura $(\mathrm{n}=1)$, and vagal nerve $(\mathrm{n}=2)$. Of the 6 patients who underwent extended resection, 5 were treated with chemotherapy. Because these patients had advanced-stage disease and the number of the subjects was small, no correlation was found between the extent of resection and the outcome.

The distribution of pathologic stage was stage IA in 19 patients $(30 \%)$, stage IB in $16(25 \%)$, stage IIA in 5 $(8 \%)$, stage IIB in $11(18 \%)$, stage IIIA in $9(14 \%)$, and stage IIIB in 3 patients $(5 \%)$. The mean MIB- 1 index for all patients was $62.7 \%$ (range, 5.2\%-90.5\%), and the mean mitotic count was 71.2/10 HPF (range, 14-153/10 HPF). All 63 patients had tumor necrosis.

Perioperative platinum-based chemotherapy was administered to $23(37 \%)$ of the 63 patients. We have used the criterion of tumor size more than $3 \mathrm{~cm}$ in offering chemotherapy for patients with stage I disease since 2004. Thus, 8 of 35 patients with stage I received chemotherapy. Also, 9 of 16 with stage II and 6 of 12 with stage III received chemotherapy. Induction chemotherapy was administered to 3 patients at clinical stage III and adjuvant chemotherapy was administered to 20 patients at clinical stage I/II. Three patients received preoperative mediastinal radiotherapy (40 Gy) combined with induction chemotherapy. No patient 
TABLE 1. Patient characteristics $(n=63)$

\begin{tabular}{|c|c|c|c|c|}
\hline Factor & Total & $\begin{array}{c}\text { Triple } \\
\text { positive }\end{array}$ & $\begin{array}{l}\text { Non-triple } \\
\text { positive }\end{array}$ & $P$ value \\
\hline Patients (n) & 63 & 31 & 32 & \\
\hline Age (y) & & & & .0473 \\
\hline Mean & 67.0 & 64.4 & 69.5 & \\
\hline Range & $30-84$ & $30-78$ & $41-84$ & \\
\hline Gender & & & & .2578 \\
\hline Male & $54(87)$ & $25(81)$ & $29(91)$ & \\
\hline Female & $9(13)$ & $6(19)$ & $3(9)$ & \\
\hline Smoking status & & & & .1512 \\
\hline Former or current & $58(92)$ & $27(87)$ & $31(97)$ & \\
\hline Never smoked & $5(8)$ & $4(13)$ & $1(3)$ & \\
\hline Surgical procedure & & & & .3416 \\
\hline Lobectomy & $55(87)$ & $26(84)$ & $29(90)$ & \\
\hline Segmentectomy & $2(3)$ & $3(10)$ & $0(0)$ & \\
\hline Wedge resection & $6(10)$ & $2(6)$ & $3(10)$ & \\
\hline Pathologic stage & & & & .6044 \\
\hline IA & $19(30)$ & $11(35)$ & $8(25)$ & \\
\hline IB & $16(25)$ & $7(23)$ & $9(28)$ & \\
\hline IIA & $5(8)$ & $3(10)$ & $2(6)$ & \\
\hline IIB & $11(18)$ & $3(10)$ & $8(25)$ & \\
\hline IIIA & $9(14)$ & $5(16)$ & $4(13)$ & \\
\hline IIIB & $3(5)$ & $2(6)$ & $1(3)$ & \\
\hline MIB-1 index (\%) & & & & .5029 \\
\hline Mean & 62.7 & 61.2 & 64.4 & \\
\hline Range & $5.2-90.5$ & $5.2-90.0$ & $5.8-90.5$ & \\
\hline Mitotic counts (/10 HPF) & & & & .3538 \\
\hline Mean & 71.2 & 64.7 & 77.9 & \\
\hline Range & $14-153$ & $14-122$ & $20-153$ & \\
\hline
\end{tabular}

underwent postoperative radiotherapy. The chemotherapy regimens are listed in Table 2.

The results of immunohistochemical staining for the 3 neuroendocrine markers are summarized in Table 3. Although the percentage of reactive cells ranged very much, the intensity of immunostaining was not so variegated for all 3 neuroendocrine markers. Of the 63 tumors, 40 $(63 \%)$ were positive for synaptophysin, $36(57 \%)$ for chromogranin A, and $59(94 \%)$ for NCAM. Finally, 31 tumors $(49 \%)$ were positive for all 3 neuroendocrine markers and $32(51 \%)$ were negative for 1 or 2 markers. The clinicopathologic characteristics and chemotherapy regimens of patients in the triple-positive group and non-triple-positive group are listed in Tables 1and 2, respectively. Although the triple-positive group was significantly younger than the non-triple-positive group, no significant differences were seen in the distribution of other characteristics between the 2 groups. Also, no morphologic differences were found between the 2 groups in the neuroendocrine structures such as rosettes and ribbon-like arrangements, necrosis, mitotic counts, and MIB-1 index.
TABLE 2. Regimens of perioperative platinum-based chemotherapy $(\mathbf{n}=\mathbf{2 3})$

\begin{tabular}{lcc}
\hline \multicolumn{1}{c}{ Regimen } & $\begin{array}{c}\text { Triple } \\
\text { positive }(\mathbf{n}=\mathbf{1 2})\end{array}$ & $\begin{array}{c}\text { Non-triple } \\
\text { positive }(\mathbf{n}=\mathbf{1 1})\end{array}$ \\
\hline Induction chemotherapy & 1 & 0 \\
CDDP + VP-16 & 0 & 1 \\
CDDP + VNR & 1 & 0 \\
CBDCA + DOC & & \\
Adjuvant chemotherapy & 5 & 2 \\
CDDP + CPT-11 & 2 & 4 \\
CBDCA + PTX & 2 & 3 \\
CDDP + VNR & 1 & 1 \\
CBDCA + VP-16 & 1 & \\
\hline
\end{tabular}

Triple positive, Positive for synaptophysin, chromogranin A, and neural cell adhesion molecule; Non-triple positive, negative for 1 or 2 neuroendocrine markers (synaptophysin, chromogranin $\mathrm{A}$, and neural cell adhesion molecule); $C D D P$, cisplatin; $V P-16$, etoposide; $V N R$, vinorelbine; $C B D C A$, carboplatin; $D O C$, docetaxel; CPT-11, irinotecan; PTX, paclitaxel.

The overall 5-year survival rate among the 63 patients was $44.9 \%$. Significantly longer survival was observed for the patients who underwent surgery with chemotherapy than for those who underwent surgery without chemotherapy $(74.4 \%$ and $32.3 \%$, respectively; $P=.042$; Figure 1 , A).

Next, we evaluated whether the effects of perioperative chemotherapy were seen in patients with different stages. Although there was a tendency for longer survival for the patients with stage I disease who underwent surgery and chemotherapy compared with those who underwent surgery without chemotherapy, the small number of subjects did not allow us to obtain a statistically significant difference $(85.7 \%$ and $35.2 \%$, respectively; $P=.1129$; Figure $1, B)$. Similarly, no statistically significant difference in survival between the patients with and without chemotherapy at stage II/III $(68.8 \%$ and $25.6 \%$, respectively; $P=.1243$; Figure $1, B)$. Multivariate analysis of survival was

TABLE 3. Immunohistochemical staining of 3 neuroendocrine markers $(n=63)$

Neuroendocrine marke

Patients (n)

Synaptophysin

Positive

$40(63)$

Negative

23 (37)

Chromogranin A

Positive

$36(57)$

Negative

$27(43)$

NCAM

Positive

$59(94)$

Negative

4 (6)

Triple positive

$31(49)$

Non-triple positive

$32(51)$

Data in parentheses are percentages. NCAM, Neural cell adhesion molecule; Triple positive, Positive for synaptophysin, chromogranin A, and neural cell adhesion molecule; Non-triple positive, negative for 1 or 2 neuroendocrine markers (synaptophysin, chromogranin A, and neural cell adhesion molecule). 
All patients $(n=63)$

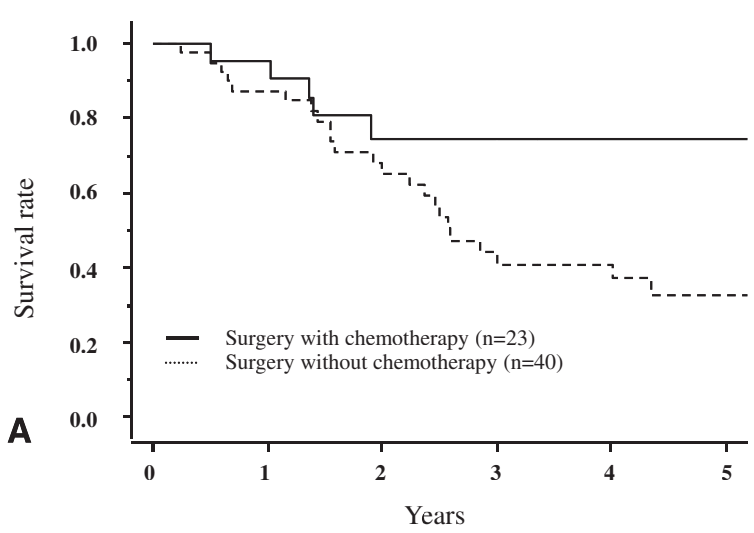

All patients $(n=63)$

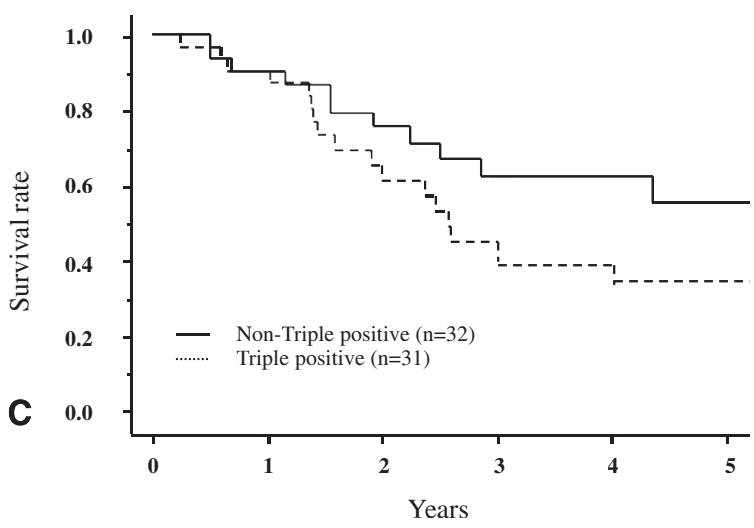

Non-triple positive patients $(n=32)$

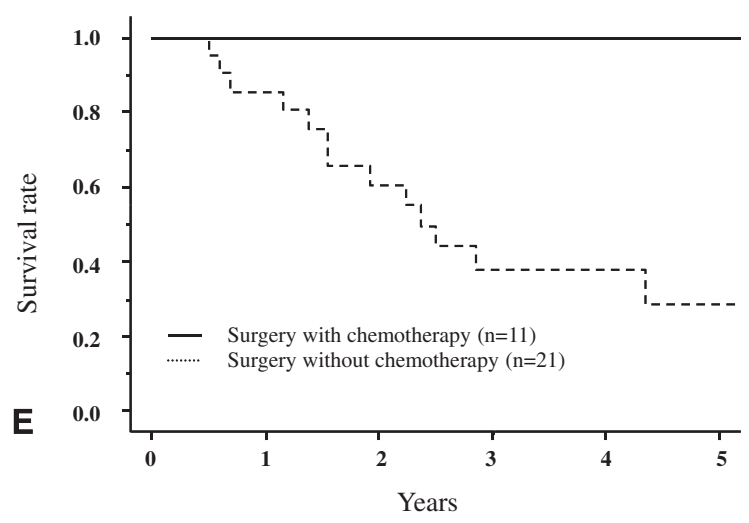

Stage specific outcome $(n=63)$

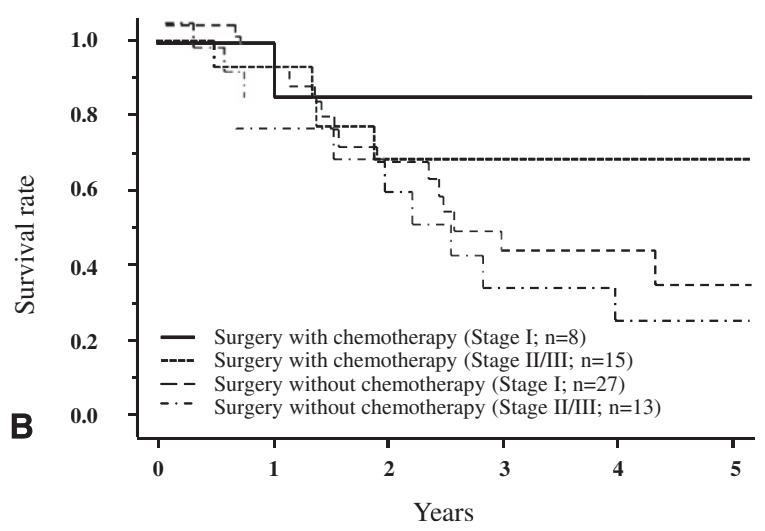

Surgery with chemotherapy $(n=23)$

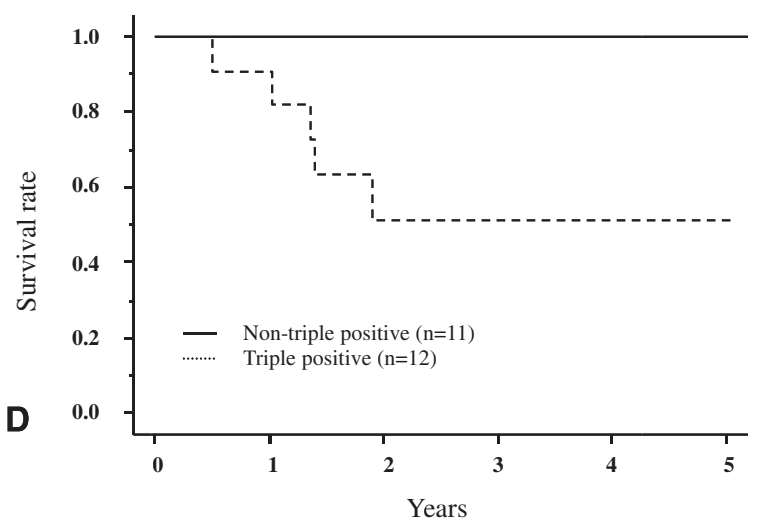

Triple positive patients $(\mathrm{n}=31)$

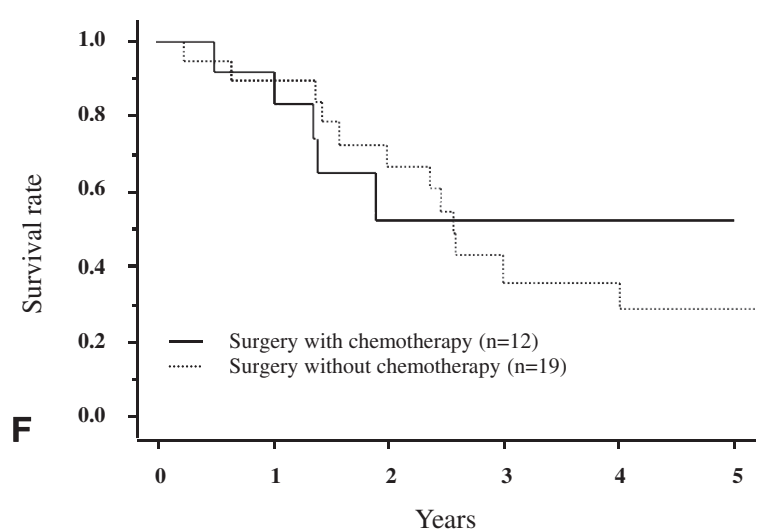

FIGURE 1. A, Comparison of survival of patients with large cell neuroendocrine carcinoma who underwent surgery with perioperative chemotherapy and those who underwent surgery alone. B, Comparison of stage-specific survival of patients with large cell neuroendocrine carcinoma who underwent surgery with perioperative chemotherapy and those who underwent surgery alone (stage I vs stage II/III). C, Comparison of survival of the non-triple-positive group and triple-positive group. D, Comparison of survival of the non-triple-positive group and triple-positive group among patients who received perioperative chemotherapy. E, Comparison of survival of non-triple-positive patients who underwent surgery with perioperative chemotherapy and those who underwent surgery without perioperative chemotherapy. F, Comparison of survival of triple-positive patients who underwent surgery with perioperative chemotherapy and those who underwent surgery without perioperative chemotherapy. Non-triple positive, Negative for 1 or 2 neuroendocrine markers (synaptophysin, chromogranin A, and neural cell adhesion molecule); Triple positive, positive for synaptophysin, chromogranin A, and neural cell adhesion molecule. 
TABLE 4. Multivariate analysis of prognostic factors and survival (Cox proportional hazards model)

\begin{tabular}{lccc}
\hline \multicolumn{1}{c}{ Variable } & HR & $\mathbf{9 5} \%$ CI & $\boldsymbol{P}$ value \\
\hline Age $(<75$ vs $\geq 75$ y) & 1.030 & $0.466-2.279$ & .9409 \\
Gender (male vs female) & 1.091 & $0.400-2.967$ & .8659 \\
Pathologic stage (I vs II/III) & 0.645 & $0.286-1.455$ & .2904 \\
$\begin{array}{l}\text { Surgical procedure (lobectomy vs } \\
\quad \text { sublobar resection) }\end{array}$ & 1.048 & $0.287-3.824$ & .9431 \\
$\begin{array}{l}\text { Treatment (surgery with } \\
\text { chemotherapy vs surgery alone) }\end{array}$ & 0.323 & $0.112-0.934$ & .0371 \\
\hline
\end{tabular}

$H R$, Hazard ratio; $C I$, confidence interval.

performed using 5 clinical prognostic factors (age, gender, pathologic stage, surgical procedure, and surgery with or without chemotherapy; Table 4). Patients who underwent surgery with chemotherapy had a significantly better prognosis than those who underwent surgery without chemotherapy (hazards ratio, $0.323 ; 95 \%$ confidence interval, $0.112-0.934 ; P=.0371$ ).

Next, we examined whether the clinical outcome of patients with LCNEC correlated with the immunohistochemical characteristics determined by immunoreactivity for 3 neuroendocrine markers. No significant difference was found in 5-year survival between the triple-positive and non-triple-positive patients $(34.0 \%$ and $55.3 \%$, respectively; $P=.1312$; Figure $1, C$ ). No statistically significant difference was found in survival among the singlepositive, double-positive, and triple-positive patients (data not shown). Among the patients who received perioperative chemotherapy, a significantly greater 5 -year survival rate was observed in the non-triple-positive group than in the triple-positive group $(100 \%$ and $51.9 \%$, respectively; $P=.0216$; Figure $1, D)$. Moreover, in the non-triple-positive group, a significantly greater survival rate at 5 years was observed in patients who underwent surgery with adjuvant chemotherapy than in those who underwent surgery without chemotherapy $(100 \%$ and $34.5 \%$, respectively; $P=.0081$; Figure $1, E$ ). In contrast, in the triple-positive group, no difference was found in 5-year survival between the patients who underwent surgery with adjuvant chemotherapy and those who underwent surgery without chemotherapy $(51.8 \%$ and $28.1 \%$, respectively; $P=.7682$; Figure $1, F)$.

We further analyzed the correlation of chemotherapy benefits and immunoreactivity patterns of neuroendocrine markers in patients with different stages. The patients with stage I and stage II/III did not differ in overall survival in the non-triple-positive group $(53.2 \%$ and $56.3 \%$, respectively; $P=.8910$; Figure 2, A). Survival differences were also not found in the triple-positive group between stage I and stage II/III $(36.8 \%$ and $28.2 \%$, respectively; $P=.6460 ;$ Figure 2, $B$ ).

Because a limited number of patients with stage I disease received perioperative chemotherapy, we failed to show a statistically significant survival difference between the patients with and without chemotherapy in the non-triplepositive patients $(100 \%$ and $40.6 \%$, respectively; $P=.2002$; Figure $2, C$ ) and the triple-positive patients $(80 \%$ and $25.2 \%$, respectively; $P=.2606$; Figure $2, D)$. However, perioperative chemotherapy resulted in a significantly greater 5-year survival rate in the non-triple-positive group patients with stage II/III than in the triple-positive group $(100 \%$ and $17.9 \%$, respectively; $P=.0074$; Figure 2,E). No correlation was found between the use of perioperative chemotherapy and the survival of patients with stage II/III disease in the triple-positive group. In the group of patients with triple-positive tumors, the 5-year survival rate of the patients with chemotherapy and without chemotherapy was $34.3 \%$ and $33.3 \%$, respectively $(P=.6108 ;$ Figure $2, F)$.

\section{DISCUSSION}

Neuroendocrine lung tumors comprise a spectrum of epithelial neoplasms ranging from low-grade carcinoid tumor to SCLC. Although most SCLCs show neuroendocrine differentiation on immunohistochemistry or electron microscopy, ${ }^{13}$ a significant minority of NSCLCs (approximately $10 \%-30 \%$ ) show neuroendocrine differentiation. NSCLCs with neuroendocrine differentiation are considered to result in an especially poor prognosis. Several reports have indicated that NSCLCs with neuroendocrine differentiation were clinically aggressive with greater chemosensitivity; however, other studies have not shown any correlation. ${ }^{8,14}$ A 5-year survival rate of $15 \%$ to $57 \%$ has been reported for all stages of LCNEC. ${ }^{10,11}$ Sarkaria and colleagues ${ }^{11}$ reported a 5-year survival rate of $37 \%$ for patients with stage IB-IIIA LCNEC who did not receive perioperative platinum-based chemotherapy compared with $51 \%$ in those patients who received it. Saji and colleagues ${ }^{10}$ reported that the 5-year survival rate for patients undergoing perioperative chemotherapy was $87.5 \%$ and that of patients without perioperative chemotherapy was $58.5 \% .{ }^{10}$ Our results were similar.

Thus, we assumed that pulmonary LCNEC might have several features that make it sensitive to chemotherapy and tried to evaluate the association between the 3 neuroendocrine markers that are essential for the diagnosis of LCNEC and the responsiveness to chemotherapy. Positive immunostaining for 1 or more neuroendocrine markers among synaptophysin, chromogranin A, and NCAM is necessary to diagnose pulmonary LCNEC. Synaptophysin is a synaptic vesicle glycoprotein with 4 transmembrane domains; however, its exact function is unknown. ${ }^{15}$ Chromogranin $\mathrm{A}$ is the major member of the granin family of acidic secretory glycoproteins and plays multiple roles in the secretory process. ${ }^{16} \mathrm{NCAM}$, a glycoprotein, is a member of the immunoglobulin superfamily and contributes to the function of cell-cell adhesion. ${ }^{17}$ Although all 3 markers are present in neuroendocrine cells, it remains possible 


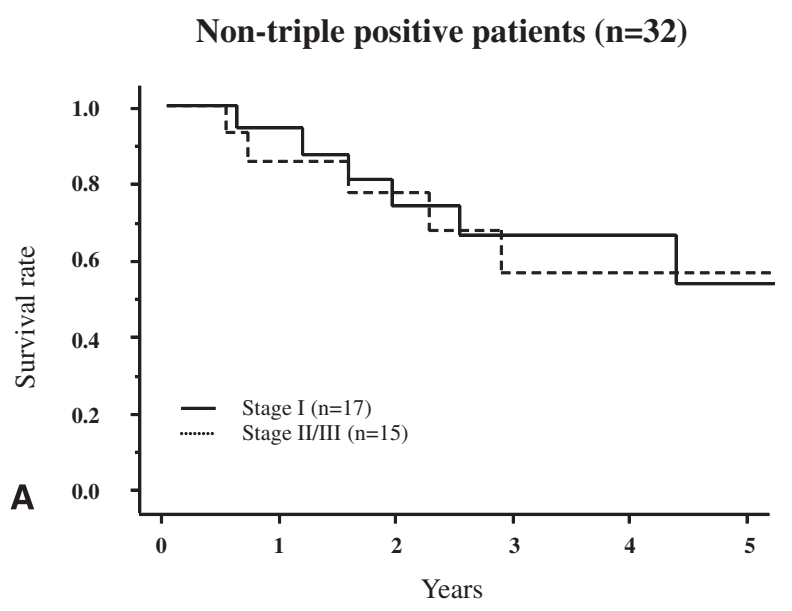

Non-triple positive patients with stage I ( $n=17)$

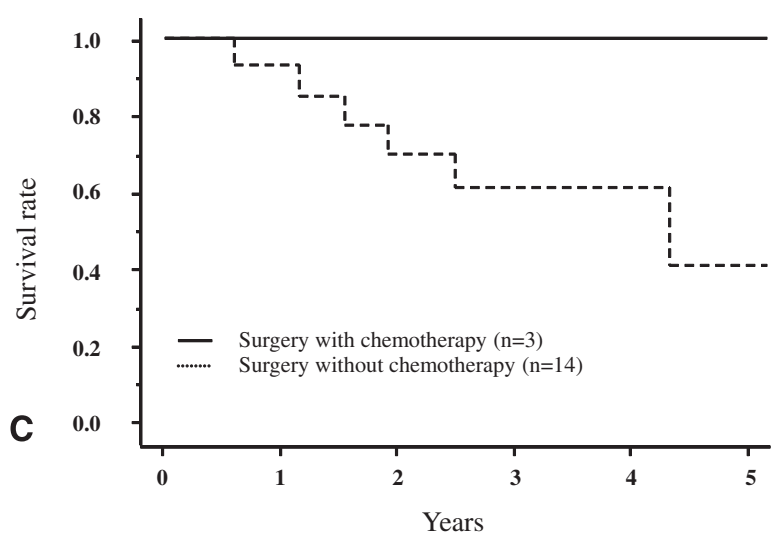

Non-triple positive patients with stage II/III $(n=15)$

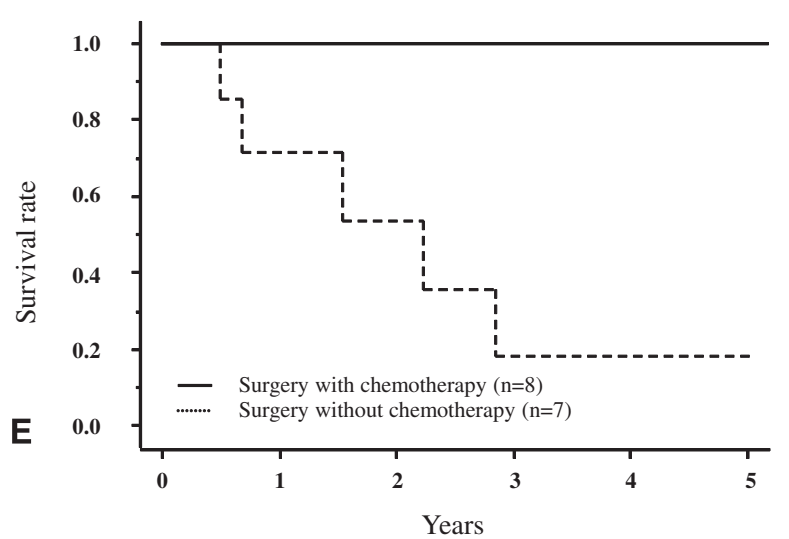

Triple positive patients $(n=31)$

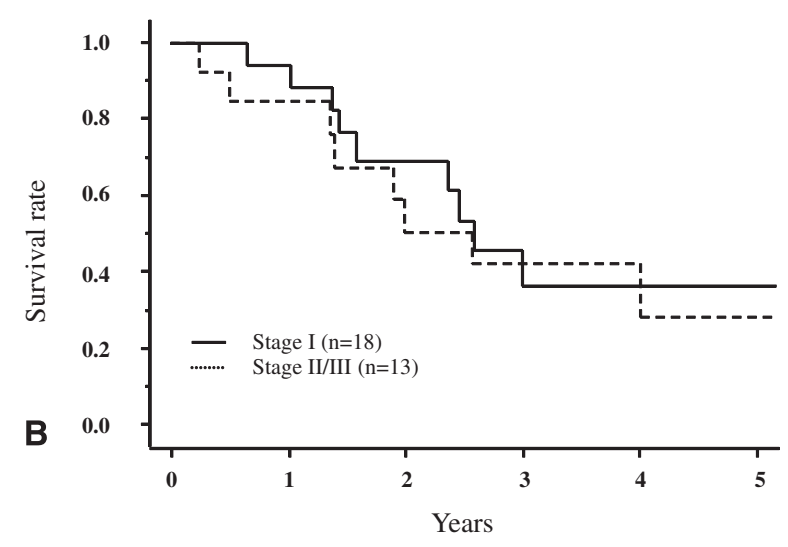

Triple positive patients with stage $I(n=18)$

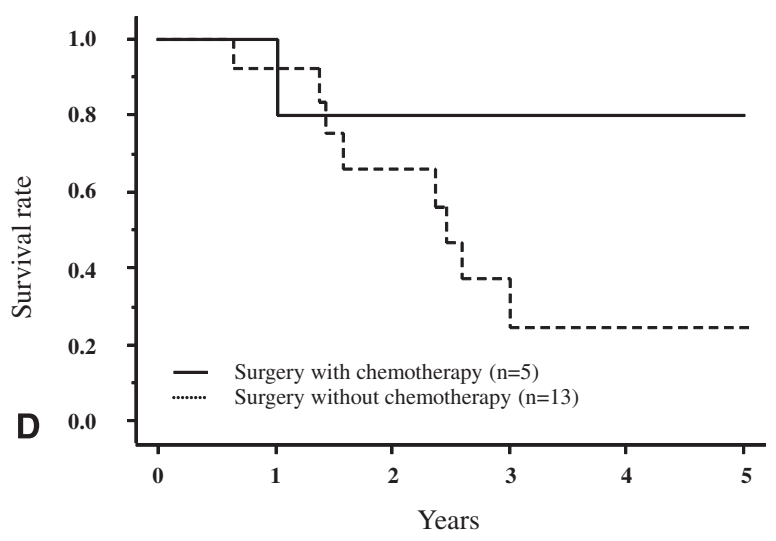

Triple positive patients with stage II/III (n=13)

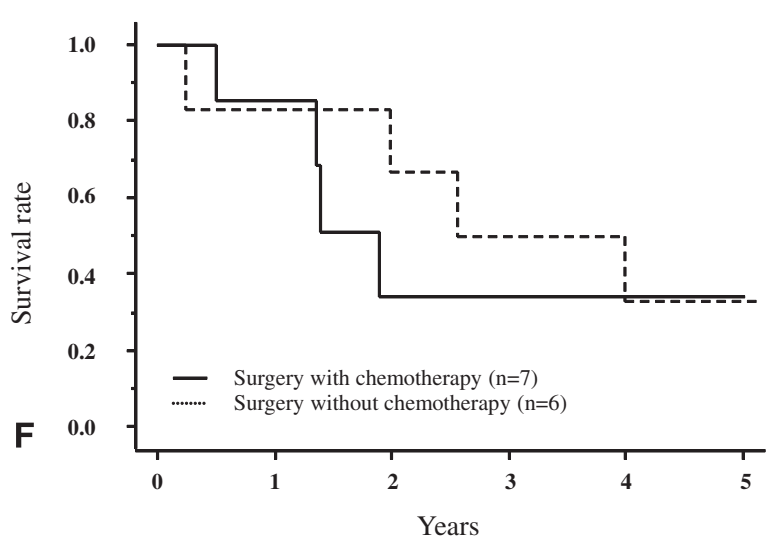

FIGURE 2. A, Comparison of stage-specific survival of patients with non-triple-positive large cell neuroendocrine carcinoma. B, Comparison of stage-specific survival of patients with triple-positive large cell neuroendocrine carcinoma. C, Comparison of survival of patients with stage I nontriple-positive disease who underwent surgery with perioperative chemotherapy and those who underwent surgery without perioperative chemotherapy. D, Comparison of survival of patients with stage I triple-positive who underwent surgery with perioperative chemotherapy and those who underwent surgery without perioperative chemotherapy. E, Comparison of survival of patients with stage II/III non-triple-positive disease who underwent surgery with perioperative chemotherapy and those who underwent surgery without perioperative chemotherapy. F, Comparison of survival of patients with stage II/III triple-positive disease who underwent surgery with perioperative chemotherapy and those who underwent surgery without perioperative chemotherapy. Non-triple positive, Negative for 1 or 2 neuroendocrine markers (synaptophysin, chromogranin A, and neural cell adhesion molecule); Triple positive, positive for synaptophysin, chromogranin A, and neural cell adhesion molecule. 
that LCNEC does not have all 3 proteins. It was reported that neuroendocrine markers are often negative in poorly differentiated neuroendocrine cancers, ${ }^{18}$ and it is estimated that LCNEC in the non-triple-positive group tended to be poorly differentiated and associated with a poor prognosis. Our results have demonstrated that the 5-year survival rate of patients who did not undergo perioperative chemotherapy in the non-triple-positive group was $34.5 \%$. The addition of perioperative chemotherapy improved the prognosis of LCNEC in the non-triple-positive group but did not improve the prognosis of LCNEC in the triple-positive group. We considered that LCNEC might become resistant to chemotherapy through coexistence and mutual interaction of synaptophysin, chromogranin A, and NCAM and might lose the ability to resist because of the deficiency of the mutual interaction, owing to a lack of any of the 3 proteins.

Various studies have analyzed LCNEC's prognostic factors using other immunohistochemical staining and gene expression profiles. ${ }^{19-21}$ However, no report has more clearly demonstrated the sensitivity of pulmonary LCNEC to perioperative chemotherapy than has our study. Moreover, it is also considered valuable that we can describe this result with 3 well-known biomarkers that are necessary for the diagnosis of pulmonary LCNEC. Furthermore, it was reported that carcinoids that exhibit good prognosis have a low response rate to chemotherapy and SCLCs that show a poor prognosis have a high initial response rate to chemotherapy. ${ }^{18,22}$ Considered with our results, it is likely that triplepositive LCNEC was rich in neuroendocrine character, similar to carcinoids, and the non-triple-positive LCNECs were poor in neuroendocrine character, similar to SCLCs.

In the present study, antibody staining was designated as negative when none of the tumor cells were stained and as positive when any degree of immunoreactivity was found. We determined the cutoff value using the following scoring system: 0 , no positive cells; $1+$, less than $10 \%$ of cells positive; $2+, 10 \%$ to $50 \%$ of cells positive; and $3+$, more than $50 \%$ of cells positive. From this analysis, the optimal cutoff, defined as the value that best separated a poor prognostic group from a better prognostic group, was nonimmunoreactive (negative) vs immunoreactive (positive) for the neuroendocrine markers. The evaluation separating "positive" from "negative," without any counting of cells, was easily and quickly performed with high reproducibility, which could be an advantage in possible future use in the clinical setting.

At present, most LCNECs are diagnosed using surgically resected specimens and rarely using biopsy or cytology specimens. Almost all publications concerning resected LCNEC have been based on the retrospective analyses of surgical specimens. ${ }^{23} \mathrm{We}$ used postoperative specimens to diagnose pulmonary LCNEC and to categorize them as either triple positive or non-triple positive. However, it would be difficult to categorize LCNEC according to our criteria using small biopsy specimens or cytologic specimens because heterogeneity and focal and scattered positivity of immunostaining against the neuroendocrine markers are not unusual. Therefore, this method might not be applicable for neoadjuvant chemotherapy.

Regarding the perioperative chemotherapy regimens for pulmonary LCNEC, platinum-based regimens that include etoposide (VP-16) or irinotecan (CPT-11), which are standard for SCLC, are more effective than other platinumbased regimens for NSCLCs, because pulmonary LCNEC is genetically and immunohistochemically more similar to SCLC than to NSCLC. ${ }^{10,24,25}$ In our study, $10(43 \%)$ of the 23 patients underwent a platinum-based regimen that included VP-16 or CPT-11. In addition, 3 (27\%) of 11 patients in the non-triple-positive group received a platinumbased regimen that included VP-16 or CPT-11, in contrast to $7(58 \%)$ of 12 patients in the triple-positive group. We considered that our result (ie, the sensitivity of LCNEC to perioperative chemotherapy in the non-triple-positive group), was not affected by the regimen of chemotherapy that included VP-16 or CPT-11.

Evidence is increasing that surgical resection alone is insufficient as treatment of LCNEC, even for stage I disease, and perioperative platinum-based chemotherapy might provide a survival advantage for patients with stage I LCNEC. ${ }^{9,10}$ Our results have demonstrated that patients with stage I LCNEC tended to benefit from perioperative chemotherapy, although we failed to demonstrate a significant difference because only a small number of patients with stage I received perioperative chemotherapy. In the patients with stage I, perioperative chemotherapy tended to be associated with longer survival in the non-triple-positive group, as well as in the triple-positive group.

Although we acknowledge our study's limitations (a small number of subjects and short-term follow-up), our results have demonstrated that perioperative chemotherapy can enhance survival for the patients in the non-triple-positive group, although no correlation was seen between chemotherapy and survival in the triple-positive group. We believe these preliminary results are a reasonable rationale for a larger study to determine the correlation between chemotherapy response and neuroendocrine immunoreactivity in patients with LCNEC.

\section{CONCLUSIONS}

Our results have suggested that perioperative chemotherapy can be an important therapeutic option in the treatment of pulmonary LCNEC, particularly in the non-triple-positive patients. In the future, prospective multi-institutional studies with larger sample sizes should be conducted to verify the validity of our findings. Continued studies, including molecular studies, are also important to further improve the treatment stratification of patients with LCNEC. 


\section{References}

1. Travis WD, Linnoila RI, Tsokos MG, Hitchcock CL, Cutler GB Jr, Nieman L, et al. Neuroendocrine tumors of the lung with proposed criteria for large-cell neuroendocrine carcinoma: an ultrastructural, immunohistochemical, and flow cytometric study of 35 cases. Am J Surg Pathol. 1991;15:529-53.

2. Travis WD, Corrin B, Shimosato Y. Histological typing of lung and pleural tumors. In: World Health Organization International Histological Classification of Tumors. 3rd ed. Berlin: Springer-Verlag; 1999.

3. Cooper WA, Thourani VH, Gal AA, Lee RB, Mansour KA, Miller JI. The surgical spectrum of pulmonary neuroendocrine neoplasms. Chest. 2001;119:14-8.

4. Iyoda A, Hiroshima K, Toyozaki T, Haga Y, Fujisawa T, Ohwada H. Clinical characterization of pulmonary large cell neuroendocrine carcinoma and large cell carcinoma with neuroendocrine morphology. Cancer. 2001;91: 1992-2000.

5. Takei H, Asamura H, Maeshima A, Suzuki K, Kondo H, Niki T, et al. Large cell neuroendocrine carcinoma of the lung: a clinicopathologic study of eighty-seven cases. J Thorac Cardiovasc Surg. 2002;124:285-92.

6. Battafarano RJ, Fernandez FG, Ritter J, Meyers BF, Guthrie TJ, Cooper JD, et al. Large cell neuroendocrine carcinoma: an aggressive form of non-small cell lung cancer. J Thorac Cardiovasc Surg. 2005;130:166-72.

7. Asamura H, Kameya T, Matsuno Y, Noguchi M, Tada H, Ishikawa Y, et al. Neuroendocrine neoplasms of the lung: a prognostic spectrum. J Clin Oncol. 2006; 24:70-6.

8. Iyoda A, Hiroshima K, Toyozaki T, Haga Y, Baba M, Fujisawa T, et al. Adjuvant chemotherapy for large cell carcinoma with neuroendocrine features. Cancer. 2001;92:1108-12.

9. Iyoda A, Hiroshima K, Moriya Y, Takiguchi Y, Sekine Y, Shibuya K, et al Prospective study of adjuvant chemotherapy for pulmonary large cell neuroendocrine carcinoma. Ann Thorac Surg. 2006;82:1802-7.

10. Saji H, Tsuboi M, Matsubayashi J, Miyajima K, Shimada Y, Imai K, et al. Clinical response of large cell neuroendocrine carcinoma of the lung to perioperative adjuvant chemotherapy. Anticancer Drugs. 2010;21:89-93.

11. Sarkaria IS, Iyoda A, Roh MS, Sica G, Kuk D, Sima CS, et al. Neoadjuvant and adjuvant chemotherapy in resected pulmonary large cell neuroendocrine carcinomas: a single institution experience. Ann Thorac Surg. 2011;92:1180-6.

12. Sobin L, Wittekind $\mathrm{CH}$, eds. TNM classification of malignant tumours. 6th ed. New York: Wiley-Liss; 2002. p. 99-103.

13. Guinee DG Jr, Fishback NF, Koss MN, Abbondanzo SL, Travis WD. The spectrum of immunohistochemical staining of small-cell lung carcinoma in specimens from transbronchial and open-lung biopsies. Am J Clin Pathol. 1994;102:406-14.

14. González-Aragoneses F, Moreno-Mata N, Cebollero-Presmanes M, GarcíaYuste M, Cañizares-Carretero MA, Molins-López-Rodó L, et al. Prognostic significance of synaptophysin in stage I of squamous carcinoma and adenocarcinoma of the lung. Cancer. 2007;110:1776-81.

15. Leung ASY, Cooper K, Leung FJWM. Manual of Diagnostic Antibodies for Immunohistology. London; Greenwich Medical Media: 1999:307.

16. Gosney JR, Gosney MA, Lye M, Butt SA. Reliability of commercially available immsunocytochemical markers for identification of neuroendocrine differentiation in bronchoscopic biopsies of bronchial carcinoma. Thorax. 1995;50:116-20.

17. Ionescu DN, Treaba D, Gilks CB, Leung S, Renouf D, Laskin J, et al. Nonsmall cell lung carcinoma with neuroendocrine differentiation — an entity of no clinical or prognostic significance. Am J Surg Pathol. 2007;31:26-32.

18. Gustafsson BI, Kidd M, Chan A, Malfertheiner MV, Modlin IM. Bronchopulmonary neuroendocrine tumors. Cancer. 2008;113:5-21.

19. Faggiano A, Sabourin JC, Ducreux M, Lumbroso J, Duvillard P, Leboulleux S, et al. Pulmonary and extrapulmonary poorly differentiated large cell neuroendocrine carcinomas: diagnostic and prognostic features. Cancer. 2007;110:265-74.

20. Harada M, Yokose T, Yoshida J, Nishiwaki Y, Nagai K. Immunohistochemical neuroendocrine differentiation is an independent prognostic factor in surgically resected large cell carcinoma of the lung. Lung Cancer. 2002;38:177-84.

21. Beasley MB, Lantuejoul S, Abbondanzo S, Chu WS, Hasleton PS, Travis WD, et al. The P16/cyclin D1/Rb pathway in neuroendocrine tumors of the lung. Hum Pathol. 2003;34:136-42.

22. Turrisi AT III, Kim K, Blum R, Sause WT, Livingston RB, Komaki R, et al. Twice-daily compared with once-daily thoracic radiotherapy in limited smallcell lung cancer treated concurrently with cisplatin and etoposide. $N$ Engl $J$ Med. 1999;340:265-71.

23. Travis WD, Rush W, Flieder DB, Falk R, Fleming MV, Gal AA, et al. Survival analysis of 200 pulmonary neuroendocrine tumors with clarification of criteria for atypical carcinoid and its separation from typical carcinoid. Am J Surg Pathol. 1998;22:934-44.

24. Rossi G, Cavazza A, Marchioni A, Longo L, Migaldi M, Sartori G, et al. Role of chemotherapy and the receptor tyrosine kinases KIT, PDGFRalpha, PDGFRbeta, and Met in large-cell neuroendocrine carcinoma of the lung. J Clin Oncol. 2005; 23:8774-85.

25. Rusch VW, Klimstra DS, Venkatraman ES. Molecular markers help characterize neuroendocrine lung tumors. Ann Thorac Surg. 1996;62:798-809. 710. 